\title{
Experimental Study of Nucleate Pool Boiling of FC-72 on Smooth Surface under Microgravity
}

\author{
Yan-Fang Xue • Jian-Fu Zhao • Jin-Jia Wei • Jing Li • \\ Dong Guo · Shi-Xin Wan
}

Received: 2 March 2011 / Accepted: 29 June 2011 / Published online: 12 July 2011

(C) Springer Science+Business Media B.V. 2011

\begin{abstract}
Experiments of highly subcooled nucleate pool boiling of FC-72 with dissolved air were studied both in short-term microgravity condition utilizing the drop tower Beijing and in normal gravity conditions. The bubble behavior and heat transfer of air-dissolved FC-72 on a small scale silicon chip $\left(10 \times 10 \times 0.5 \mathrm{~mm}^{3}\right)$ were obtained at the bulk liquid subcooling of $41 \mathrm{~K}$ and nominal pressure of $102 \mathrm{kPa}$. The boiling heat transfer performance in low heat flux region in microgravity is similar to that in normal gravity condition, while vapor bubbles increase in size but little coalescence occurs among bubbles, and then forms a large bubble remains attached to the heater surface during the whole microgravity period. Thermocapillary convection may be an important mechanism of boiling heat transfer in this case. With further increasing in heat flux to the fully developed nucleate boiling region, the vapor bubbles number as well as their size significantly increase in microgravity. Rapid coalescence occurs among adjacent bubbles and then the coalesced large bubble can depart from the heating surface during the microgravity period. The reason of the large bubble departure is mainly attributed to the momentum effects caused by the coalescence of small bubbles with the large one. Hence, the steady-state pool boiling can still be ob-
\end{abstract}

Y.-F. Xue · J.-J. Wei $(\bowtie) \cdot$ D. Guo

State Key Laboratory of Multiphase Flow in Power Engineering, School of Energy and Power Engineering, Xi'an Jiaotong University, Xi'an 710049, China e-mail: jjwei@mail.xjtu.edu.cn

J.-F. Zhao $\cdot$ J. Li $\cdot$ S.-X. Wan

Key Laboratory of Microgravity (National Microgravity

Laboratory) / CAS; Institute of Mechanics, Chinese

Academy of Sciences, Beijing 100190, China tained in microgravity. In the high heat flux regime near the critical heat flux, significant deterioration of heat transfer was observed, and a large coalesced bubble forms quickly and almost covers the whole heater surface, leading to the occurrence of the critical heat flux in microgravity condition.

Keywords Microgravity $\cdot$ Pool boiling $\cdot$ Heat transfer • Thermocapillary convection $\cdot$ Coalescence

\section{Introduction}

Boiling heat transfer is one of the key technologies for future space-based hardware and processes such as the cooling of electronic devices, heat exchange and the material processing and so on, due to its high heat transfer performance in comparison with single-phase heat transfer. The study of pool boiling in microgravity can provide fundamental information for the development of space boiling heat transfer devices. In addition, an understanding of nucleate boiling and critical heat flux (CHF) in microgravity environments is of importance for the safe operation of space heat exchangers. However, boiling is a very complex and illusive process where heat transfer, mass transfer, hydrodynamics and interfacial phenomena are closely interrelated.

In earth gravity, the buoyancy effects caused by the large density differences of the phase on boiling heat transfer are so dominant that weaker forces effects are masked and cannot be studied separately. Often this leads to an incorrect physical view of the process itself, and to an inadequate mathematical description, as well (Straub 2001). Advances in the understanding of boiling phenomenon have been greatly hindered 
by masking effect of gravity. Microgravity experiments offer a unique opportunity to exclude, or at least to minimize, the buoyancy effect, and to study boiling phenomena and their self-induced dynamics without the influence of external forces. Furthermore, they can also provide a means to study the actual influence of gravity on the boiling. On the other hand, since many potential applications exist in space and in planetary neighbors due to its high efficiency in heat transfer, pool boiling in microgravity has become an increasing significant subject for investigation (Zhao 2011). Therefore, the microgravity researches will be conductive to revealing of the mechanism underlying the phenomenon, and then developing of more mechanistic models for the related applications both on earth and in space. On the progress in this field, several comprehensive reviews and monographs are available now. For instance, Straub (2001) issued a comprehensive review of his own activity on this field from the early 1980s to date, while Di Marco (2003); Kim (2003); Ohta (2003); and Zhao (2010) recently issued reviews of microgravity boiling researches in Europe, in US, in Japan, and in China, respectively. Thus, only results related to the present study, namely those on nucleate pool boiling on plain plate heaters in microgravity can shortly summarized in the following.

The early work of Siegel and Keshock (1964), studied nucleate pool boiling in saturated distilled water, using a drop shaft with $1 \mathrm{~s}$ duration. They used a very smooth nickel plate as the heater surface. The results indicate that isolated bubbles in reduced gravity become large and that the growth times are long compared with those in earth gravity. Their observation also reveals that a large bubble previously detached absorbs the newly growing bubbles on the heater surface, and thus serves as a temporary vapor sink. They postulated that the absorption could increase turbulence induced near the surface, which would serve to increase the heat transfer coefficient.

Littles and Walls (1970) conducted pool boiling experiments with R113 using a drop tower which provided a duration of $4.1 \mathrm{~s}$. They used a relatively large and even wide flat copper heater $50.8 \times 101.6 \mathrm{~mm}^{2}$ in size. They found that steady-state boiling in reduced gravity can be obtained with saturated liquids. Furthermore, the boiling curve shifted upward when compared to curves obtained in earth gravity. This corresponds to an enhancement in the process.

Straub and coworkers (Zell et al. 1989; Straub et al. 1990) studied nucleate boiling with organic refrigerants R113 and R12 as working fluids; using a ballistic rocket (TEXUX program) and parabolic flights provide microgravity conditions, with a $20 \times 40 \mathrm{~mm}^{2}$ gold- coated flat plate as a heater surface. The results were confirmed that the process of boiling is not gravity dependent and the main heat transport mechanism involved is evaporation process. They also observed that in subcooled liquids the vapor bubbles remain attached to the surface, postulating that the bubbles contribute to thermocapillary flow or Marangoni convection induced along the liquid-vapor interface while the bubbles are attached to the heater surface. This is attributed to a surface tension variation with temperature at the interface.

In general, thermocapillary convection results from surface tension gradients along an interface due to temperature gradients, concentration gradients, and electrical potential variations or gradients (Ostrach 1982):

$\frac{d \sigma}{d t}=\frac{\partial \sigma}{\partial T} \frac{\partial T}{\partial t}+\frac{\partial \sigma}{\partial c} \frac{\partial c}{\partial t}+\frac{\partial \sigma}{\partial \varphi} \frac{\partial \varphi}{\partial t}$.

The thermocapillary convection may be of importance as a heat transfer mechanism in nucleate boiling, which was originally suggested by McGrew (1966), who questioned the explanation that high boiling heat transfer rate are attributed to intense vapor bubble agitation of the liquid boundary layer adjacent to the heating surface and bulk liquid entrainment by bubble detachment from the surface. They performed a series of experiments in which the liquid was heated from above and cooled from below, with slowly increasing heat flux. The results indicated that thermocapillary convection would occur around any bubble presenting in a region subjected to a temperature gradient, and the observed phenomenon served as an important factor in the heat transfer mechanism in those situations where bubbles remained attached to the surface for relatively long periods of time.

Straub and coworkers (Straub 2002; Marek and Straub 2001) also investigated the origin and effect of thermocapillary convection in subcooled nucleate pool boiling. Their results demonstrated that thermocapillary convection can form in such systems in the following manner. In subcooled boiling, the top of a growing bubble may extend out of the superheated boundary layer and start to condense. With evaporation occurring near the three phase contact line, impurities such as dissolved gas are carried along with vapor to the top of the bubble. The vapor subsequently condenses while the noncondensable gases accumulate along the interface. Under steady-state conditions, the presence of the noncondensable gas reduces the vapor pressure locally along the interface thereby decreasing the saturation temperature locally, resulting in a positive temperature gradient along the interface towards the bubble base. These temperature gradients generate along the bubble 
interface inducing thermocapillary flow from the base of the bubble to its top. In contrast, at saturated pool boiling a thermocapillary flow could never be detected.

Lee and Merte (1999) and Lee et al. (1997) conducted pool boiling experiments with R113 in space flights that provided a gravity level of $10^{-4} g_{0}$ for periods of $280 \mathrm{~s}$. A $19 \times 38 \mathrm{~mm}^{2}$ flat gold film heater was used that allowed video camera viewing both from the side and through the bottom of the heater. The results demonstrated that not only can a steady pool boiling process exist in microgravity, but an enhancement in the heat transfer occurs with both saturated and subcooled boiling. They also found that a heat transfer enhancement of up to $40 \%$ was attributed to bubble migration. Thermocapillary convection was thought to cause this kind of motion.

Kim and Benton (2002) and Kim et al. (2002) investigated systemically the effect of gravity and subcooling on the boiling heat transfer behavior during pool boiling of FC-72 on a $2.7 \mathrm{~mm} \times 2.7 \mathrm{~mm}$ microheater array using a KC-135 aircraft. Their observations indicated that the boiling heat transfer behavior is dominated by the formation of a large primary bubble on the surface by the coalescence of smaller bubbles under a low-g environment. Besides, dryout occurred under the primary bubble, causing critical heat flux (CHF) in low-g to be significantly lower then in earth gravity. Subcooling was observed to have a strong effect on the size of the primary bubble and on CHF. Furthermore studies on the effects of thermocapillary convection on boiling heat transfer from heaters with various aspect ratios during different gravity levels have also been reported by the same group, such as Henry and Kim (2005).

Zhao et al. (2009) who conducted experiments on pool boiling of degassed FC-72 on a plane plate heater $\left(28 \times 20 \mathrm{~mm}^{2}\right)$ aboard the Chinese recoverable satellite SJ-8. Their observation reveals that for high subcooling, nucleate boiling and local dry area at the base of the coalesced large bubble can co-exist. Besides, they also found that an abrupt transition to film boiling can be observed and then an obvious turning point corresponding to CHF appears on the boiling curve. It was also shown that heat transfer coefficient and CHF increase with the subcooling or pressure in microgravity, as observed in normal gravity. The value of $\mathrm{CHF}$ in microgravity, however, was only about one third of that at the similar pressure and subcooling in terrestrial condition.

Although the efforts expended have been considerable, the results appear inconclusive, with various interpretations of the mechanisms for the heat transfer in nucleate pool boiling under microgravity condition and, in some cases, contradictory experimental results. Therefore, the present paper is to study the combined effects of bubble dynamics and thermocapillary convection on nucleate pool boiling heat transfer of FC72 in short-term microgravity condition over smallscale heater surface for further observation and interpretation of the experimental phenomenon to provide some new insight into fundamental understanding of the mechanism. The experiments of highly subcooled pool boiling of FC-72 with air-dissolved on a smooth silicon chip with the dimension of $10 \times 10 \times 0.5 \mathrm{~mm}^{3}$ were developed, and a microgravity condition was provided with the gravity level less than $10^{-2} g_{0}$ (here $g_{0}=9.81 \mathrm{~m} / \mathrm{s}^{2}$ ) in a short duration of about $3.6 \mathrm{~s}$ by using the drop tower Beijing. It is ought to be pointed out that the aim of test liquid choosing air-dissolved FC-72 is to study the effect of thermocapillary convection in subcooled nucleate pool boiling in microgravity condition. Because small amounts of noncondensable gas was one of preconditions to induce thermocapillary convection (Straub 2002; Marek and Straub 2001).

\section{Experimental Apparatus and Test Procedure}

\subsection{Test Facility Description}

A simple, but useful pool boiling test facility system designed for the present drop-tower experiments is shown schematically in Fig. 1. The boiling chamber, which was made of polycarbonate for visualizing boiling phenomena and had a size of $140 \times 140 \times 155 \mathrm{~mm}^{3}$, was filled with about $3 \mathrm{~L}$ test liquid of FC-72 with air-dissolved. A rubber bag attached to the test vessel was used to maintain a nearly atmospheric pressure. Video of the bubble behavior was obtained using a $25 \mathrm{~Hz}$ CCD camera, which installed in front of the test vessel at a direction angle of $45^{\circ}$ with respect to the heater surface for visual observation. Simultaneously, a high speed digital camera (VITcam CTC) imaging 250 frames per second at a resolution of $1024 \times 640$ pixels with a shutter speed of $1 / 2000 \mathrm{~s}$ was used along with a lens (Computar MLM-3XMP) to obtain images of the boiling process. The high speed digital camera was installed in front of the test vessel at a direction angle of $30^{\circ}$ with respect to the heater surface.

A $0.13-\mathrm{mm}$ diameter T-type thermocouple for the local wall temperature $\left(T_{3}\right)$ measurement was bonded on the bottom surface of test chip at the center using epoxy adhesive. The local temperature of test liquid $\left(T_{2}\right)$ was measured by a $0.3-\mathrm{mm}$ diameter T-type thermocouple which was located at about $20 \mathrm{~mm}$ apart from the edge of the test chip and $40 \mathrm{~mm}$ above the 
Fig. 1 Schematic diagram of the experimental apparatus



chip level in order to avoid the effect of the heater. The change of measured temperature of bulk liquid is less than $0.5{ }^{\circ} \mathrm{C}$ during every test run about $90 \mathrm{~min}$. The two thermocouples for the measurements of liquid and wall temperatures were connected with a data acquisition system (DI710-UHS). The uncertainties in the chip and bulk liquid temperature measurements by the thermocouples are estimated to be less than $0.3 \mathrm{~K}$. Wall temperature uncertainty can be attributed to the errors caused by thermocouple calibration by a platinum resistance thermometer $(0.03 \mathrm{~K})$, temperature correction for obtaining surface temperature from the measured value at the bottom of the chip $(0.2 \mathrm{~K})$, the temperature unsteadiness $(0.1 \mathrm{~K})$ and the thermocouple resolution (less than $0.1 \mathrm{~K}$ ). The uncertainty of the bulk temperature is due to errors caused by thermocouple calibration by a platinum resistance thermometer $(0.03 \mathrm{~K})$, the temperature unsteadiness $(0.2 \mathrm{~K})$ and the thermocouple resolution (less than $0.1 \mathrm{~K}$ ).

A P-doped N-type square silicon chip with the dimensions of $10 \times 10 \times 0.5 \mathrm{~mm}^{3}$ with a smooth surface was used for the heater element as shown in Fig. 2. The test chip had a nominal resistance of $69 \Omega$. The specific resistance was $1-2 \Omega \mathrm{cm}$, and the thermal conductivity $k$ was about $120 \mathrm{~W} / \mathrm{mK}$. The chip was Joule heated by a direct current. Two 0.25 -mm diameter copper wires were soldered with a lower temperature solder (the melting point of $180^{\circ} \mathrm{C}$ ) to the side surfaces at the opposite ends of test chip that supply power to the chip by a direct current. In order to secure the ohmic contact between the semiconductor silicon chip and the copper wire, a special solder with the melting point of $300{ }^{\circ} \mathrm{C}$ was applied to the silicon chip with ultrasonic bonding method before soldering the copper wires. This method can be greatly reduced or negligible the contact resistance between the silicon chip and the copper wires. The test chip was bonded on a $50 \times 50 \times 1.2 \mathrm{~mm}^{3}$ Plexiglass plate using epoxy adhesive and then fixed on the Plexiglass base which is bonded on the bottom of the test vessel. This facilitated an easy exchange of

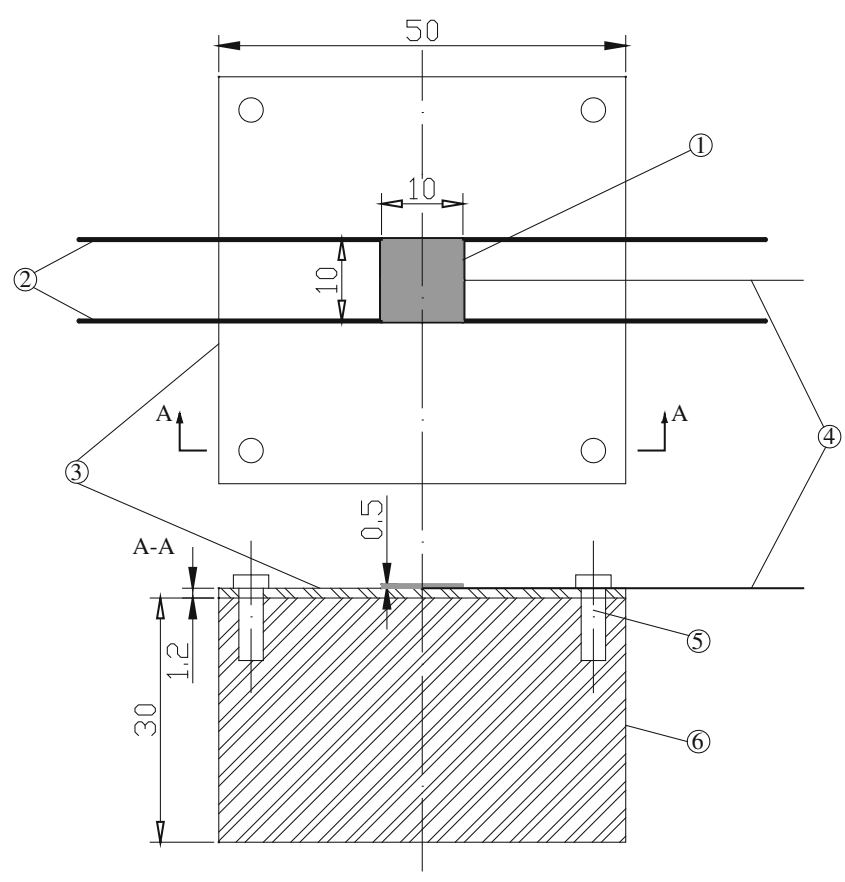

Fig. 2 Details of test section 
the silicon chip and minimization of conduction heat loss. The side surfaces of the chip were covered with an adhesive to minimize heat loss. Therefore, only the upper surface of the chip was effective for heat transfer because the heat loss to the side and base surfaces of silicon chip are neglected. A programmable DC power supply was connected in parallel to two series resistances $\left(R_{1}=5 \mathrm{k} \Omega, R_{2}=100 \mathrm{k} \Omega\right)$ and the test chip. The two series resistances were used to measure the voltages across the silicon chip in the circuit. Thus, the heating power was calculated by constant current and measured voltage across the test chip. Besides, according to the measurement of heater surface temperature, the inner energy stored in the test chip itself can be calculated. At last, the heat flux from the heating surface to liquid can be calculated by subtracting the increase of the inner energy of the test chip from the input heat flux. Heat flux uncertainty includes the error of electric power supplied to the chip $(0.11 \%)$, which is calculated from the errors of the current $(0.014 \%)$ and voltage $(0.1 \%)$ across the chip and heat loss by substrate heat conduction and copper lead wires. The heat loss was estimated by solving three-dimensional conduction problems through substrate using a commercial software PHONICS, which was less than 5\% in the nucleate boiling heat transfer region. Furthermore, the difference of heat loss is small for both in normal gravity and microgravity conditions. Detail analyses of the uncertainty were done by Wei (2002).

\subsection{Test Procedure}

Experiments were conducted both in terrestrial gravity and in short-term microgravity in the drop tower Beijing in the National Microgravity Laboratory, Chinese Academy of Sciences. The facility satisfied the safety criteria to expose the apparatus in the drop capsule to different gravitational environments varying between microgravity ( $\mu g$; less than $10^{-2} g_{0}$ ) in the free falling period and high-g level in the deceleration recovery system $\left(16 g_{0}\right)$.

The test chip was heated by setting a constant input current for the desired heat flux $q_{\text {desired }}$ to initiate boiling on the heater surface. After the heat transfer reached a steady state in about $2 \mathrm{~min}$, the free falling of drop capsule started which could provide approximately $3.6 \mathrm{~s}$ effective microgravity environment. The high-speed digital camera could work for a duration time of $4 \mathrm{~s}$ to get the boiling heat transfer phenomena after an external trigger signal was received. Moreover, the data measurement and the video recording were operated simultaneously.
Experiments were performed in five test runs at five different heat fluxes, for which the heating currents are $0.20,0.24,0.30,0.36$ and $0.40 \mathrm{~A}$, respectively. The working fluid was FC-72 at about $102 \mathrm{kPa}$ pressure. Only one liquid subcooling was tested in the present experiment due to limited experiment opportunity. As the first step, the aim of this study is to clarify the experimental phenomenon and some basic mechanisms. Thus, the liquid subcooling keeps at about $41 \mathrm{~K}$ (corresponding to $14.6{ }^{\circ} \mathrm{C}$ of bulk liquid temperature) which is caused by ambient temperature. The mole fraction of the airdissolved concentration in FC-72 was measured by a gas chromatograph, which was about 2600 3300 ppm. Identical ground experiment was also conduct for comparison. It should be pointed out that the identical ground test experiment did not achieve the critical heat flux in the present study to avoid damage to the test chip.

\section{Results and Discussion}

The transition of vapor bubble behavior and the mean heater surface temperature (denoted as Chip S) responding to the variation of gravity level for the heating dc current of $0.20,0.24,0.30$ and $0.36 \mathrm{~A}$ (the corresponding heat flux range from 3.38 to $11.59 \mathrm{~W} / \mathrm{cm}^{2}$ ) are shown in Figs. 3 and 4, respectively. The positions of the Fig. 3 for different heating currents are correspondingly marked on the curves of the mean temperature of Chip $\mathrm{S}$ shown in Fig. 4. The liquid subcooling keeps at about $41 \mathrm{~K}$ (corresponding to $14.6{ }^{\circ} \mathrm{C}$ of bulk liquid temperature). The time for entering the microgravity condition is set to $0 \mathrm{~s}$. It can be seen that before entering the microgravity condition, according to the moving videos the bubbles generate and departure continuously from the heating surface at a steady state due to the effects of buoyancy forces (as shown in Fig. 3 at -1.2 s). However, after entering microgravity condition, due to the weakness of the buoyancy force, the vapor bubbles generate and attach to the heater surface for a longer time and then grow to a larger size than under terrestrial condition (as shown in Fig. 3 at $0.2 \mathrm{~s}$ ). At the same time, the mean heater surface temperature remains nearly constant for the heating dc current of $0.20,0.24$, and 0.30 A shown in Fig. 4 (such as $T_{\mathrm{w}}(1), T_{\mathrm{w}}(2)$, and $\left.T_{\mathrm{w}}(3)\right)$ regardless of the variation of the bubbles behavior and the effect of gravity reduction. This demonstrated that even the weakness of buoyancy force does not decrease the heat transfer in microgravity condition at low heat fluxes. For single phase heat transfer, the natural convection heat transfer caused by buoyancy force play an important role. With the bubble generation at 
Fig. 3 Bubble behavior at developing and fully developed nucleate boiling regimes under different gravity levels. Corresponding to the curves of the Fig. 4



$-1.2 \mathrm{~s} T_{\mathrm{w}}(1)=49.3^{\circ} \mathrm{C}$

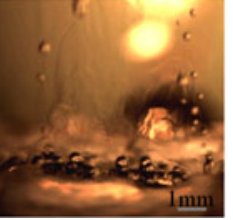

$-1.2 \mathrm{~s} T_{\mathrm{w}}(2)=54.3^{\circ} \mathrm{C}$



$-1.2 \mathrm{~s} T_{\mathrm{w}}(3)=58.3^{\circ} \mathrm{C}$

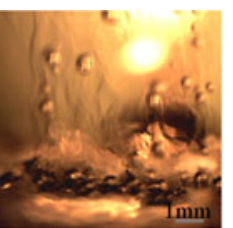

$-1.2 \mathrm{~s} T_{\mathrm{w}}(4)=61.4^{\circ} \mathrm{C}$

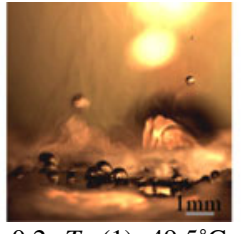

$0.2 \mathrm{~s} T_{\mathrm{w}}(1)=49.5^{\circ} \mathrm{C}$

(1) $I=0.20 \mathrm{~A}$

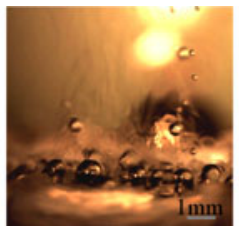

$0.2 \mathrm{~s} T_{\mathrm{w}}(2)=54.6^{\circ} \mathrm{C}$

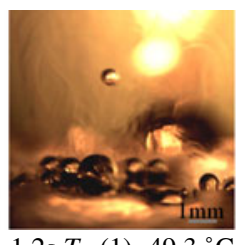

$1.2 \mathrm{~s} T_{\mathrm{w}}(1)=49.3^{\circ} \mathrm{C}$

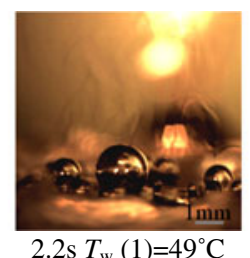

$2.2 \mathrm{~s} T_{\mathrm{w}}(1)=49^{\circ} \mathrm{C}$

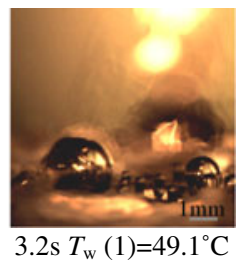

(2) $I=0.24 \mathrm{~A} \quad q_{\mathrm{ave}}=4.74 \mathrm{~W} / \mathrm{cm}^{2}$



$0.2 \mathrm{~s} T_{\mathrm{w}}(3)=58.7^{\circ} \mathrm{C}$

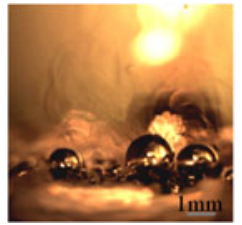

$1.2 \mathrm{~s} T_{\mathrm{w}}(2)=54.3^{\circ} \mathrm{C}$

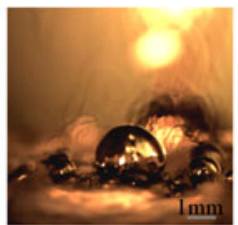

$2.2 \mathrm{~s} T_{\mathrm{w}}(2)=53.8^{\circ} \mathrm{C}$

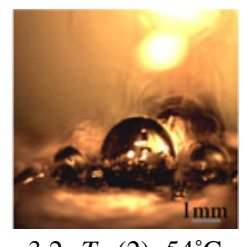

$3.2 \mathrm{~s} T_{\mathrm{w}}(2)=54^{\circ} \mathrm{C}$

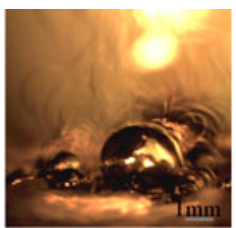

$1.2 \mathrm{~s} T_{\mathrm{w}}(3)=59.3{ }^{\circ} \mathrm{C}$

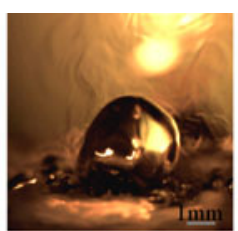

$2.2 \mathrm{~s} T_{\mathrm{w}}(3)=60.2^{\circ} \mathrm{C}$

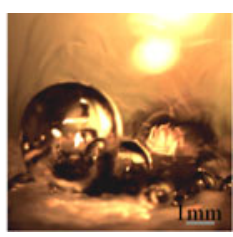

$3.2 \mathrm{~s} T_{\mathrm{w}}(3)=60.1^{\circ} \mathrm{C}$

(3) $I=0.30 \mathrm{~A} \quad q_{\mathrm{ave}}=7.42 \mathrm{~W} / \mathrm{cm}^{2}$

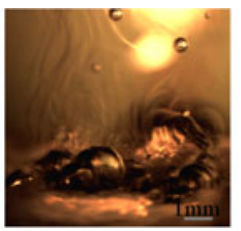

$0.2 \mathrm{~s} T_{\mathrm{w}}(4)=61.8^{\circ} \mathrm{C}$

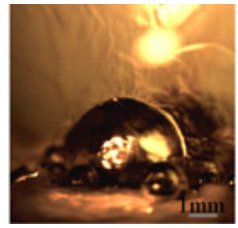

$1.2 \mathrm{~s} T_{\mathrm{w}}(4)=64.4^{\circ} \mathrm{C}$

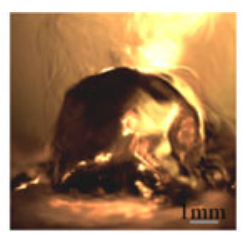

$2.2 \mathrm{~s} T_{\mathrm{w}}(4)=68.4^{\circ} \mathrm{C}$

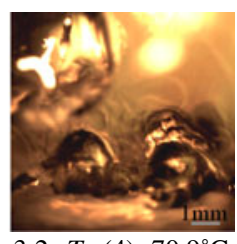

$3.2 \mathrm{~s} T_{\mathrm{w}}(4)=70.9^{\circ} \mathrm{C}$

(4) $I=0.36$ A $\quad q_{\mathrm{ave}}=11.59 \mathrm{~W} / \mathrm{cm}^{2}$

low heat fluxes of boiling process, the heat transfer is dominated by the evaporation heat transfer process of liquid through the bubble interface and enough liquid supply for evaporation, which is much more important than natural convection caused by buoyancy force. Furthermore, Lee (2002) pointed out that for organic fluids with subcooled boiling, evaporation near the bottom of the vapor bubble takes place at an elevated saturation temperature because of the local vapor recoil taking place, whereas condensation near the top of the bubble occurs at a reduced saturation temperature due to the local pressure drop associated with the condensation. Thus, a strong thermocapillary flow, also called Marangoni convection, along the vapor bubbles interface is induced from the superheated thermal liquid boundary layer to the subcooled bulk liquid. This is somewhat similar to the operation of a heat pipe. The bubbles are isolated at low heat fluxes, the large space between bubbles and then the thermocapillary convection caused by the temperature gradient along the bubble interface can guarantee the easy access of fresh bulk liquid to the heater surface for the heat transfer. Therefore, the fresh liquid can be continuously supplied beneath the bubbles attached to the heater surface, presumably due to the natural convection caused by the residual buoyancy and to the microconvection caused by temperature gradient along the bubbles surfaces but the former gradually decreases and the latter gradually increases after entering microgravity condition. With increasing time up to $1.2 \mathrm{~s}$, at a little higher heat flux (as shown in Fig. 3 at $q_{\text {ave }}=7.42 \mathrm{~W} / \mathrm{cm}^{2}$ ), a large bubble develops that is larger than other bubbles with nearly uniform size. The large bubble does not depart, remains at the surface and grows by evaporation from the thin superheated liquid layer adjacent to the heater surface. The thermocapillary convection causes the reaction force or recoil pressure that exerted on the bubble interface, which presses the bubble to the heater surface for a longer period (as shown in Fig. 5). Additionally, another observation was made that the surrounding 


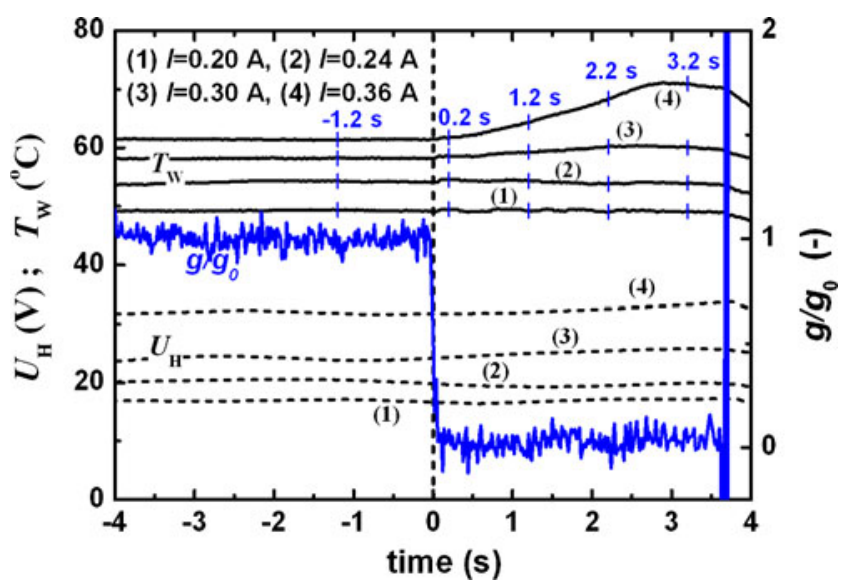

Fig. 4 Variations of the heating voltage and the surface temperature for developing and fully developed nucleate boiling regions under different gravity levels

smaller bubbles appear to be not attached to the heater surface by the thermocapillary reaction force. It can be speculated that up to the height of the small bubbles the liquid boundary layer is nearly at saturation temperature, thus the small bubbles grow up in saturated liquid. These small bubbles are freely movable from the heater surface and they slide toward the large bubble and coalesce with it. This bubble behavior is also observed by Lee (2002) and defined as bubble migration with a measured velocity of approximately $2.5 \mathrm{~cm} / \mathrm{s}$ under conditions of high subcooling at low heat fluxes. Furthermore, in normal gravity, a slipping motion was also observed by Duursma et al. (2011) for pool boiling of FC-72 on a thin wire. This motion may be caused by the capillary action in a thin liquid microlayer between the vapor bubble interface and the heater surface, also called micro-wedge model, as shown schematically in Fig. 6. Then, the large bubble attached to the heater surface continuously absorbs the small bubbles on the

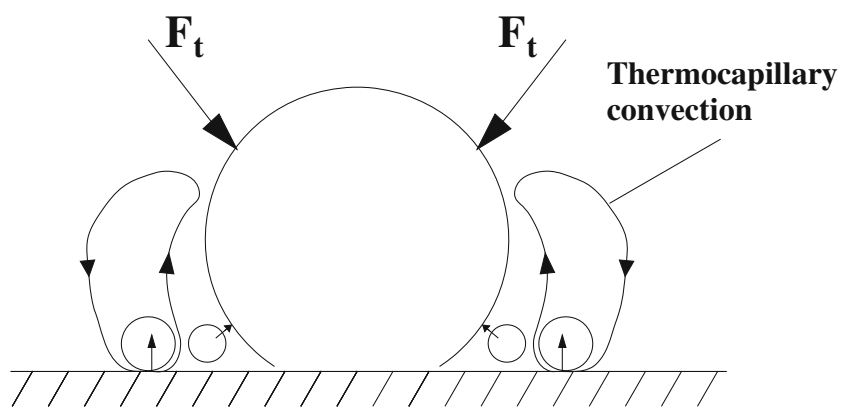

Heater

Fig. 5 Schematic diagram of the mechanism of thermocapillary convection; $F_{t}$ refers to reaction force caused by thermocapillary convection

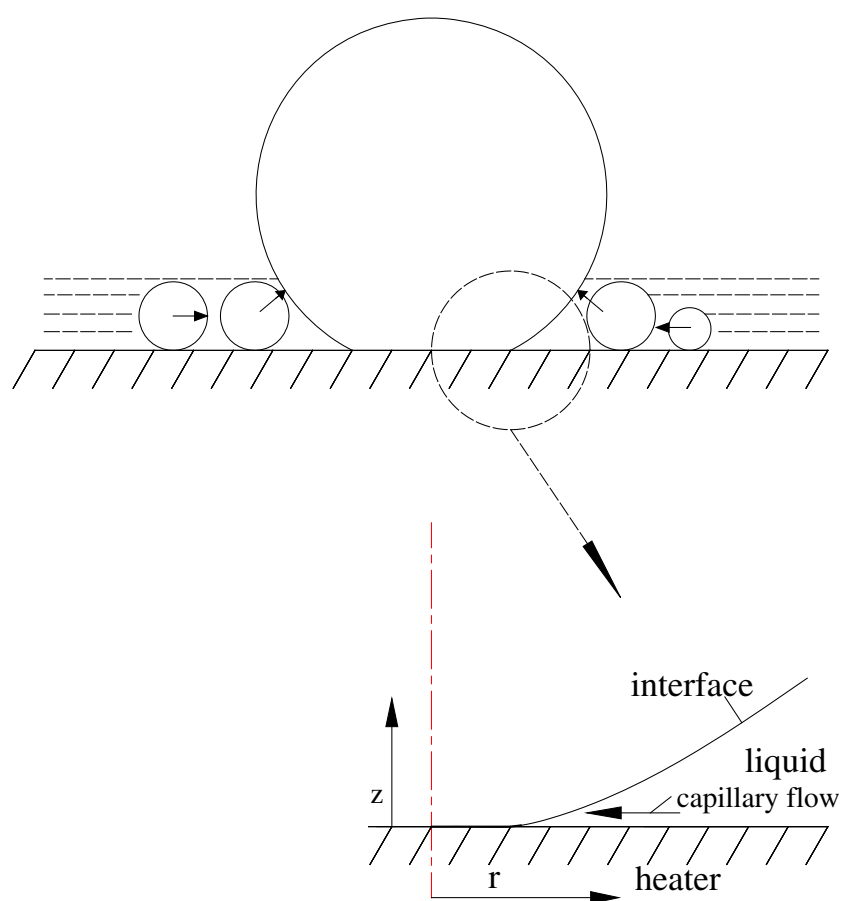

Fig. 6 Schematic diagram of micro-wedge model underneath a large bubble

heater surface and forms a larger vapor bubble, which remains attached to the heater surface throughout the entire microgravity period. However, the steady-state nucleate pool boiling can be obtained in microgravity condition. It can be postulated that, in the low heat flux region (called as developing nucleate boiling regime), the thermocapillary convection around the bubble on the heater surface plays an important role in the heat transport from the heater surface to the bulk liquid, which carries the warm condensed liquid away from the vapor-liquid interface and transports cold liquid from the bulk to the vapor-liquid interface.

With further increasing in heat flux to the fully developed nucleate boiling region (as shown in Fig. 3 at $q_{\text {ave }}=11.59 \mathrm{~W} / \mathrm{cm}^{2}$ ), the vapor bubbles number as well as their size significantly increase in microgravity. Likewise, the mean temperature of Chip S (as shown in Fig. 4 for $\left.T_{\mathrm{w}}(4)\right)$ increases slightly as time elapse in microgravity condition until it reaches its maximum peak and simultaneously adjusts itself to a quasi-steady state. This is probably due to rapid coalescence occurs among adjacent bubbles and occasionally the base of the large bubble may be dryout partially, and in the end the coalesced large bubble can depart from the heating surface during the microgravity period (as shown in Fig. 3 at $q_{\text {ave }}=11.59 \mathrm{~W} / \mathrm{cm}^{2}, 3.2 \mathrm{~s}$ ). As a consequence, the reason of the large bubble departure is mainly attributed to the vigorous lateral and vertical coalescence 
Fig. 7 Boiling behavior near the critical heat flux (CHF) under different gravity levels. Corresponding to the curves of the Fig. 8



(a) $-1.2 \mathrm{~s}$

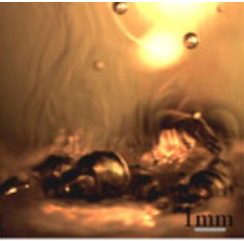

(b) $0.2 \mathrm{~s}$

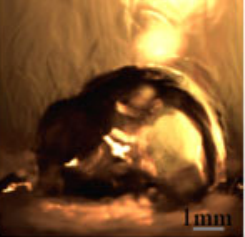

(c) $1.2 \mathrm{~s}$

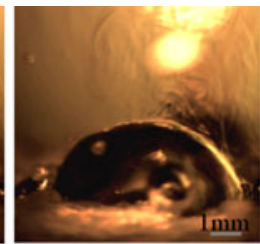

(d) $2.2 \mathrm{~s}$

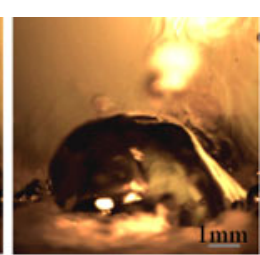

(e) $3.2 \mathrm{~s}$ processes and to the oscillation due to coalescence of adjacent bubbles and also to the momentum and inertia of liquid flow during bubble dynamic growth. Besides, it is also probably due to the thermocapillary flow generated from neighboring bubbles, caused by temperature gradients along the surface of the neighboring bubbles still attached to the heater surface.

In high heat flux regime, the influence of microgravity on the boiling heat transfer becomes more significant, and the bubble behavior is considerably different from the developing and fully developed nucleate boiling regions. Figures 7 and 8 show the variations of the bubble behavior and the mean heater surface temperature, respectively, near the critical heat flux (CHF) under different gravity levels. The positions of Figs. 7a-e are marked on the curve of the mean heater surface temperature shown in Fig. 8. It can be clearly seen that vapor bubbles experience very remarkable change with the reduction of gravity and the increase of time in microgravity. With increasing time up to $1.2 \mathrm{~s}$, a coalesced large bubble forms quickly and tends to maintain a hemispherical dome shape with a large contact area on the heater surface, causing continuously increase in wall temperature as shown in Fig. 8. With further increasing time up to $2.2 \mathrm{~s}$, the large bubble gradually shrinks to an oblate in shape and smooth in contour due to the highly subcooled condensation and covers the heater surface completely in microgravity. This is probably attributed to the higher levels of subcooling, resulting in the strong condensation at the bubble cap. And then, the oblate hemisphere bubble increases slightly in size with further increase in heating time (as shown in Fig. 7(e) $3.2 \mathrm{~s}$ ). The full coverage of the large bubble on the heater surface prevents the fresh bulk liquid from accessing the heating surface for evaporation heat transfer, making the removal of heat flux very difficult, and leading to an obvious increase of the mean heater surface temperature and deterioration of boiling heat transfer, as is observed in Fig. 8 . Furthermore, the wall temperature exceeds the upper limit cutoff of the thermocouple instrument near the end of the short-term microgravity. It is possible for the occurrence of local dryout or transition to film boiling at the bottom of the coalesced large bubble.
Figure 9 plots the boiling curves obtained in different gravity levels. There are 5 runs obtained in the short-term microgravity/drop tower experiments at five different heat fluxes respectively. Due to the limited experimental opportunity, only one run was conducted for one heat flux. The last data point with the highest heat flux is an unsteady state due to the short duration of microgravity, and then a dashed line was used to connect it with the other data points. Besides, a dashed line with arrow was also marked to indicate a transition path from normal gravity to microgravity. As a result, the true steady- or quasi-steady nucleate pool boiling can be obtained at only first four times, whereas the last exhibited a transient state boiling or film boiling associated with various degrees of dry out. Furthermore, the ground experiments with the same hardware and software were made following the first drop tower test for comparison. For the ground experiments, an agreement is quite evident, which warrants reasonable confidence in the data. The pressure and subcooling of the test conditions are about $102 \mathrm{kPa}$ and $41 \mathrm{~K}$ (corresponding to $14.6{ }^{\circ} \mathrm{C}$ of bulk liquid temperature which is caused by ambient temperature). It is pointed out that the identical ground test experiments did not get the critical heat flux point in order not to burn out the silicon chip

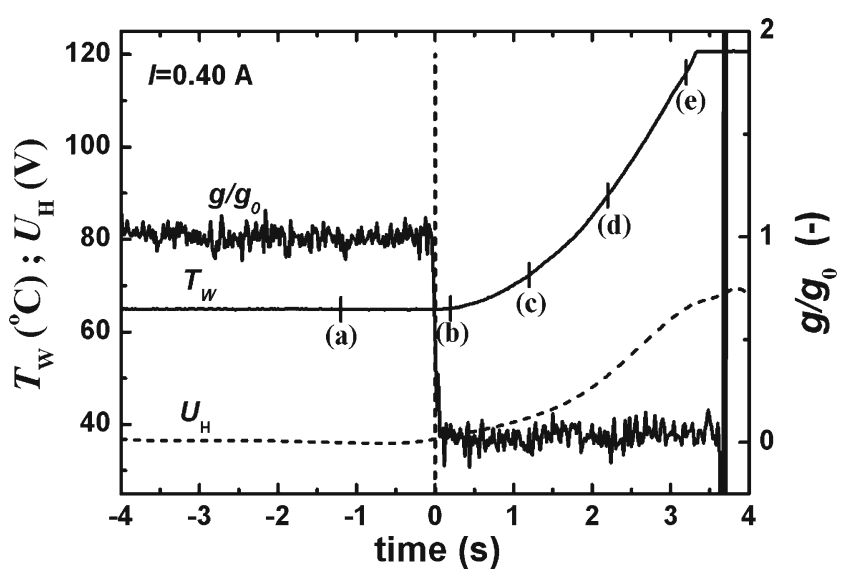

Fig. 8 Variations of the heating voltage and the surface temperature for high heat flux region under different gravity levels 


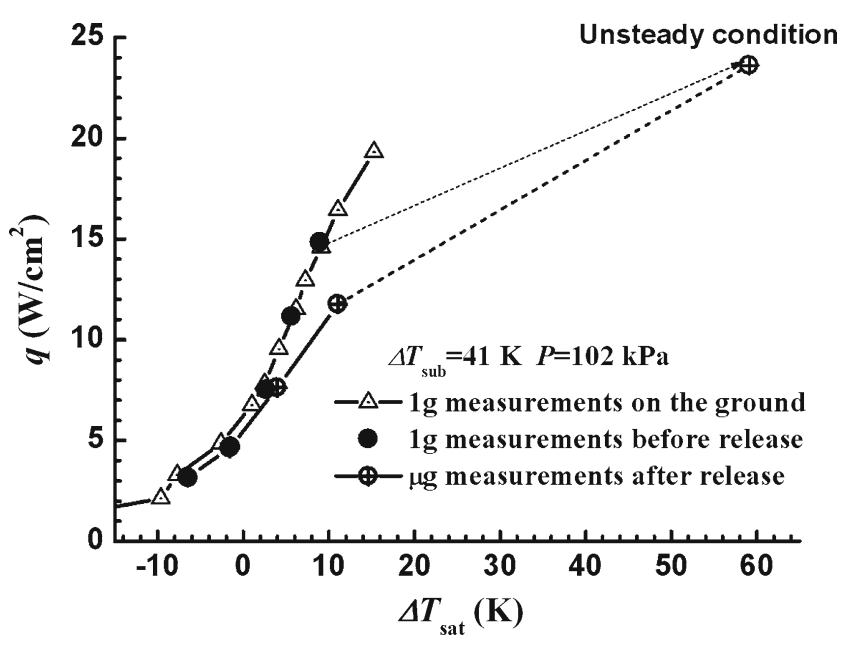

Fig. 9 Boiling curves in different gravity levels

for the following continuous microgravity drop tower experiments. Besides, it is found that the boiling starts at a negative value of $\Delta T_{\text {sat }}$ in the low heat flux region between normal gravity and microgravity conditions. Here, the wall superheat was defined as generally used in literature, i.e. $\Delta T_{s a t}=T_{w}-T_{\text {sat }}\left(P_{s}\right)$, where $P_{s}$ is the system total pressure. This is due to the effect of air-dissolved in the liquid of FC-72, which decreases the true saturation temperature of liquid, resulting in a negative value of wall superheat at the boiling incipience. In addition, as the effect of non-condensable gas on boiling curve, a quantitative compare had been made in our ground experiments (Wei 2002; Wei and Honda 2003; Honda and Wei 2004) and reveals that the existence of the gas-dissolved reduces the boiling incipient wall superheat and increases the heat flux in low heat flux region, while the effect of gas-dissolved on nucleate boiling region at high heat fluxes can be ignored.

The heat transfer in the low heat flux region for normal gravity and microgravity cases are almost exactly consistent in Fig. 9. Thus, the heat transfer in low heat flux value is independent of gravity, or at least, dependent very weakly on gravity. The vapor bubbles increase in size in microgravity, whereas remains attached to the heater surface throughout the entire microgravity period may be due to a strong thermocapillary convection effect for developing nucleate pool boiling regime. Thence, the steady state nucleate pool boiling can be maintained in microgravity.

With further increase in the heat flux, the slope of the heat transfer curve in microgravity is obviously decreased and the mean temperature of the heater surface is slowly increased compared with that in normal gravity. This is because the vapor bubbles number as well as their size significantly increase in microgravity. Moreover, coalescence occurs continuously among adjacent bubbles which become vigorous and frequent, and the coalesced large bubble continuously engulfs small bubbles around it, acting as a reservoir, and in the end the coalesced large bubble can depart from the heating surface during the microgravity period. The vigorous lateral and vertical coalescence processes, the oscillation due to coalescence of adjacent bubbles and also the momentum and inertia of liquid flow during bubble dynamic growth are the primary reasons of bubble departure in the regime of fully developed nucleate pool boiling. Thus, the steady state nucleate pool boiling can still be maintained for intermediate heat flux regime in microgravity.

In high heat flux regime, however, much evident deterioration of heat transfer can be observed in microgravity in Fig. 8. In addition, it is found that the mean heater surface superheat in microgravity condition is substantially larger than that in normal gravity. The result indicates that the deterioration of boiling heat transfer due to the reduction in gravity is significant in high heat flux region. Besides, the reason for the general increase in the mean heater surface temperature is that the coalesced large bubble with large contact area on the heater surface can not depart from the heater surface and gradually dryout at the bottom of the coalesced large bubble in microgravity condition. The occurrence of CHF or transition to film boiling is unclear. But, the trend of the increasing heater temperature with the heating time (as shown in Fig. 8) can provide some important information for the occurrence of $\mathrm{CHF}$ and dryout.

\section{Conclusion}

Bubble behavior and boiling curves for highly subcooled pool boiling of FC-72 with air-dissolved were obtained in short-term microgravity environment in the Drop Tower Beijing/NMLC. The identical ground experiments were also made following the first drop tower test for comparison.

At low heat fluxes in microgravity, the vapor bubbles increase in size but little coalescence occurs among bubbles, and the coalesced large bubble remains attached to the heating surface during the whole microgravity duration period. Besides the shortage of buoyancy, the reason for this may be the fact that there is a strong thermocapillary convection around the bubble interface due to the effect of temperature gradient, and this reacts to press the bubble toward the heater surface. 
However, the steady state nucleate pool boiling can be obtained, and the thermocapillary convection is an important mechanism of all secondary mechanisms for subcooled boiling heat transfer in microgravity.

As the heat flux increases, the vapor bubbles number as well as their size significantly increase in microgravity. Small vapor bubbles will be sucked into the large bubble through microflow caused by capillary force of the bubble interface near the heater wall. Coalescence occurs continuously among adjacent bubbles, and then the coalesced large one can depart from the heating surface mainly due to the momentum effects induced by intensive coalescence process. Hence, the steady-state pool boiling can still be obtained in microgravity.

At high heat fluxes, a coalesced large bubble forms quickly and almost completely covers the heater surface, followed by shrinking to an oblate in shape and smooth in contour due to the highly subcooled condensation. It is possible to form a local dryout on the large bubble base due to the shortage of fresh liquid supply to the heater surface. Thus, the deterioration of boiling heat transfer becomes more significant in microgravity in high heat flux regime.

\section{Nomenclature}

\begin{tabular}{|c|c|}
\hline$q$ & heat flux, $\mathrm{W} / \mathrm{cm}^{2}$ \\
\hline$q_{\text {ave }}$ & $\begin{array}{l}\text { the average value of heat flux for heater } \\
\text { surface, } \mathrm{W} / \mathrm{cm}^{2}\end{array}$ \\
\hline$U_{\mathrm{H}}$ & heating voltage, $\mathrm{V}$ \\
\hline$g$ & $\begin{array}{l}\text { gravitational acceleration at the microgravity } \\
\text { condition, } \mathrm{m} / \mathrm{s}^{2}\end{array}$ \\
\hline$g_{0}$ & $\begin{array}{l}\text { gravitational acceleration at the terrestrial } \\
\text { condition, } \mathrm{m} / \mathrm{s}^{2}\end{array}$ \\
\hline$T_{\mathrm{w}}$ & wall temperature, ${ }^{\circ} \mathrm{C}$ \\
\hline$T_{\text {sat }}$ & saturation temperature, ${ }^{\circ} \mathrm{C}$ \\
\hline$T_{b}$ & temperature of bulk liquid, ${ }^{\circ} \mathrm{C}$ \\
\hline$\Delta T_{\text {sat }}$ & wall superheat $=T_{\mathrm{w}}-T_{\mathrm{sat}}, \mathrm{K}$ \\
\hline$\Delta T_{\text {sub }}$ & liquid subcooling $=T_{\text {sat }}-T_{\mathrm{b}}, \mathrm{K}$ \\
\hline$P_{\mathrm{s}}$ & the system total pressure, $\mathrm{Pa}$ \\
\hline \multicolumn{2}{|c|}{ Greek symbols } \\
\hline$\sigma$ & surface tension, $\mathrm{N} / \mathrm{m}$ \\
\hline$\phi$ & electrical potential, $\mathrm{V}$ \\
\hline
\end{tabular}

Acknowledgements We gratefully acknowledge the financial support from the National Natural Science Foundation of China under the grants of 50806057 and 10972225, and the support from the Key Laboratory of Microgravity/CAS for experiments utilizing the drop tower Beijing. This work is also supported by the program for new century excellent talents in university (NCET-07-0608).

\section{References}

Di Marco, P.: Review of reduced gravity boiling heat transfer: european research. J. Jpn. Microgravity Appl. 20(4), 252-263 (2003)

Duursma, G.R., Jiang, F., Sefiane, K., Duff, S., Beji, H.: On the effects of thermocapillary driven oscillations on bubble growth during boiling of FC-72 on a thin wire. Int. J. Therm. Sci. (2011). doi:10.1016/j.ijthermalsci.2011.01.020

Henry, C.D., Kim, J.: Thermocapillary effects on low-g pool boiling from microheater arrays of various aspect ratio. Microgravity Sci. Technol. 16(1-4), 170-175 (2005)

Honda, H., Wei, J.J.: Enhanced boiling heat transfer from electronic components by use of surface microstructures. Exp. Therm. Fluid Sci. 28, 159-169 (2004)

Kim, J.: Review of reduced gravity boiling heat transfer: US research. J. Jpn. Microgravity Appl. 20(4), 264-271 (2003)

Kim, J., Benton, J.: Highly subcooled pool boiling heat transfer at various gravity levels. Int. J. Heat Fluid Flow 23, 497-508 (2002)

Kim, J., Benton, J., Wisniewski, D.: Pool boiling heat transfer on small heaters: effect of gravity and subcooling. Int. J. Heat Mass Transfer 45, 3919-3932 (2002)

Lee, H.S.: Mechanisms of steady-state nucleate pool boiling in microgravity. Ann. N. Y. Acad. Sci. 974, 447-462 (2002)

Lee, H.S., Merte, H.: Pool boiling mechanisms in microgravity. In: Proceedings of the International Conference on Microgravity Fluid Physics and Heat Transfer held at Oahu, Hawaii, 19-24 September, pp. 126-135 (1999)

Lee, H.S., Merte, H., Chiaramonte, F.: Pool boiling curve in microgravity. J. Thermophys. Heat Transf. 11(2), 216-222 (1997)

Littles, J.W., Walls, H.A.: Nucleate pool boiling of freon 113 at reduced gravity levels. In: Symposium on the role of nucleation in boiling and cavitation, Detroit, MI, USA, pp. 26-27 May, ASME (1970)

Marek, R., Straub, J.: The origin of thermocapillary convection in subcooled nucleate pool boiling. Int. J. Heat Mass Transfer 44, 619-632 (2001)

McGrew, J.L., Bamford, F.L., Rehm, T.R.: Marangoni flow: an additional mechanism in boiling heat transfer. Science 153, 1106-1107 (1966)

Ohta, H.: Review of reduced gravity boiling heat transfer: Japanese research. J. Jpn. Soc. Microgravity Appl. 20(4), 272-285 (2003)

Ostrach, S.: Natural convection heat transfer in cavities and cells. In: Proceedings of the Seventh International Heat Transfer Conference, Washington, DC, Hemisphere Publishing Corp, (1982)

Siegel, R., Keshock, E.G.: Effects of reduced gravity on nucleate boiling bubble dynamics in saturated water. AIChE J. 10(4), 509-517 (1964)

Straub, J.: Boiling heat transfer and bubble dynamics in microgravity. Adv. Heat Transf. 35, 57-172 (2001)

Straub, J.: Origin and effect of thermocapillary convection in subcooled boiling observations and conclusions from experiments performed at microgravity. Ann. N. Y. Acad. Sci. 974, 348-363 (2002)

Straub, J., Zell, M., Vogel, B.: Pool boiling in a reduced gravity field. In: Proc. 9th Int. Heat Transfer Conf., Jerusalem, Israel (1990) 
Wei, J.J.: Experimental study on enhanced boiling heat transfer from silicon chips with micro-pin-fins and submicron-scale roughness. PhD dissertation, Kyushu University (2002)

Wei, J.J., Honda, H.: Effects of fin geometry on boiling heat transfer from silicon chips with micro-pin-fins immersed in FC-72. Int. J. Heat Mass Transfer 46, 4059-4070 (2003)

Zell, M., Straub, J., Vogel, B.: Pool boiling under microgravity. PCH, Physicochem. Hydrodyn. 11(5/6), 813-823 (1989)
Zhao, J.F.: Two-phase flow and pool boiling heat transfer in microgravity. Int. J. Multiphase Flow 36(2), 135-143 (2010)

Zhao, J.F.: Nucleate pool boiling in microgravity. In: Belmiloudi, A. (eds.) Heat Transfer-Theoretical Analysis, Experimental Investigations and Industrial Systems, pp. 215-234 (2011)

Zhao, J.F., Li, J., Yan, N., Wang, S.F.: Bubble behavior and heat transfer in quasi-steady pool boiling in microgravity. Microgravity Sci. Technol. 21, S175-S183 (2009) 